\title{
CANCER PREVENTION BY DIET AND EXERCISE
}

Julie Anne Mitchell

Cancer Prevention Unit

NSW Cancer Council

\section{Bruce Armstrong \\ Cancer Research and Registers Division \\ NSW Cancer Council}

This article describes dietary factors for which there is strong evidence of an effect on cancer risk, and introduces the NSW Cancer Council's dietary guidelines for the prevention of cancer.

\section{BACKGROUND}

Environmental factors are thought to be responsible for some 70 per cent of human cancers. Several lines of evidence support this position. Variation in cancer risk around the world is such that if the risk of each cancer in all populations were equal to that in the lowest rate population the world burden of cancer would fall by about 70 per cent. That risk of cancer reduces in people who migrate from high-rate areas to low-rate areas also suggests that much of the worldwide variation in cancer rates is environmental in origin. Studies of Scandinavian twins are congruent in suggesting that less than 30 per cent of cancer risk is solely genetic in origin.

In a 1981 review, Doll and Peto estimated-very approximately - that 35 per cent of cancer deaths in the United States were potentially avoidable by diet modification. ${ }^{1}$ They thus suggested that diet is the major contributor to cancer caused by environment. Since that time, understanding of the relationship between diet and cancer has grown, but estimates of the contribution of diet to the burden of cancer have remained about the same. In 1997 and 1998 expert reviews concluded that about one-third of cancer deaths could be prevented by dietary change. ${ }^{2-3}$

\section{WHAT CANWE BE REASONABLY CERTAIN ABOUT?}

Dietary factors for which there is the strongest evidence of an effect on cancer risk are:

- insufficient intake of vegetables, fruit, and wholegrain cereals

- high intake of alcohol

- factors related to energy balance.

The last category does not relate to high-energy intake, but to large body size, and to lack of exercise.

\section{Vegetables and Fruit}

Of all dietary factors that contribute to cancer risk, a low intake of vegetables and fruit is the best established and probably the most important. There is convincing evidence that diets high in fruit and vegetables protect against cancers of the mouth, pharynx, oesophagus, stomach and lungs, and they probably also protect against cancers of the larynx, pancreas, breast and bladder. There is convincing evidence too that a diet high in vegetables reduces colorectal cancer risk. ${ }^{2}$

On the whole, the evidence for a protective effect of vegetables is stronger than that for a protective effect of fruits. This may reflect the greater amounts of vegetables in most diets and, perhaps, the limited range of fruits available in some populations. On a global scale, increasing intake of vegetables and fruit to optimal levels could reduce cancer incidence by about 20 per cent. ${ }^{2}$ In Australia, it has been estimated that insufficient fruit and vegetable consumption-less than five serves a dayaccounts for about nine per cent of cancer deaths. ${ }^{4}$

\section{Cereals}

Epidemiological evidence that wholegrain cereals reduce cancer risk is less persuasive than that for vegetables and fruit. Their potential to decrease bowel cancer risk, however, continues to receive attention. Components of wholegrain cereals that are thought to be protective include dietary fibre, resistant starch, antioxidants and phytoestrogens. In 1997 the World Cancer Research Fund concluded: 'diets high in wholegrain cereals possibly decrease the risk of stomach and colon cancer'. ${ }^{2}$

Part of the complexity in determining the beneficial effect of cereals relates to problems of definition. In 1970, Burkitt proposed that fibre-rich diets offered protection against numerous gastrointestinal diseases including cancer of the large bowel. Subsequent studies failed to confirm this suggestion and, in response, attention was turned to investigating key components of dietary fibre and their effect on the bowel. This has yielded valuable information on the soluble and insoluble elements of non-starch polysaccharides as well as the specific actions of resistant starch. However, it has also highlighted that all forms of dietary fibre may not be equally protective against colorectal cancer. Further to this, there is concern that in searching for the 'magic bullet' of dietary fibre, the protective effect of wholegrain cereals has not been adequately examined. ${ }^{5}$ NHMRC guidelines advise that although vegetables provide more consistent protection against colorectal cancer than cereals, patients should include poorly soluble cereal fibres in their diet, especially if they are at high risk of colorectal cancer. ${ }^{6}$

\footnotetext{
Alcohol

Alcohol consumption is a well-established cause of cancers of the upper aero-digestive tract (mouth, pharynx, larynx and oesophagus), especially in people who also smoke tobacco. It also causes cancer of the liver, probably through causing cirrhosis, and cancer of the breast. ${ }^{2}$

Risk of cancer caused by alcohol consumption increases with increasing intake right from the lowest levels. There is, therefore, no 'safe level' of alcohol intake when it comes
} 
to cancer risk. In contrast, risk of coronary heart disease initially falls as alcohol intake increases but then rises with further increase in intake. The relationship of death from all causes with alcohol intake reflects this pattern with the mortality rate in drinkers reaching that in nondrinkers at about four standard drinks a day in men and two in women.

\section{Physical Activity}

There is convincing evidence that physical activity reduces the risk of colon cancer, and it may reduce the risk of lung and breast cancers. ${ }^{2}$ The evidence is strongest for the equivalent of moderate exercise (for example, brisk walking or cycling) for an hour a day or vigorous exercise (for example, running or swimming) for half an hour a day.

\section{Body weight}

The prevalence of obesity in Australia is increasing at one per cent a year. High-risk groups are men 25-40 and women 45-55 years of age, people on a low-income, and sedentary workers. ${ }^{7}$ Evidence that obesity increases endometrial cancer is convincing. It may also contribute to renal cancer and postmenopausal breast cancer. ${ }^{2}$ To avoid obesity it is recommended that we reduce our intake of energy dense foods and nutrients (for example, fat) and increase physical activity.

\section{DIETARY GUIDELINES}

The NSW Cancer Council has developed five guidelines for a diet that will minimise cancer risk. These guidelines are designed for adults of all ages in reasonably good health. They may be changed or modified in the light of new research findings. However, while they offer no guarantee against cancer to an individual, there is strong evidence that their widespread adoption would reduce cancer incidence and mortality rates.

By way of explanation, several supporting statements are made under each guideline.

\section{Enjoy a balanced diet rich in vegetables and fruits}

The level of protection fruit and vegetable consumption provides is dependent on dose. The World Cancer Research Fund recommended five or more serves (about 400 grams) of fruit and vegetables a day for a sizeable cancer protective effect. The Cancer Council supports the NSW Health Charge through your day initiative by recommending that adults should aim to eat five serves of vegetables and two serves of fruit every day. A serve is roughly half a cup.

While some studies have suggested that particular groups of vegetables or fruits-for example, cruciferous (cabbage, broccoli, brussels sprouts), allium (onions, leeks, garlic), and green leafy vegetables; and citrus fruits-are particularly beneficial, there is insufficient evidence to support recommendations regarding specific vegetables.
Evidence that diets high in carotenoids and Vitamin $\mathrm{C}$ protect against certain cancers should not be taken as evidence that supplements of these or other micronutrients will reduce cancer risk. In the case of beta-carotene, intervention trials have shown that short-term use in highrisk individuals can increase risk of lung cancer. The emphasis of this and other guidelines is on the importance of whole foods rather than individual micronutrients.

\section{Eat a variety of wholegrain cereals, breads and pastas}

Adults should eat a minimum of seven and preferably more serves of cereals each day. A serve is equivalent to a bread roll or a cup of wholegrain cereal.

At this time there is insufficient evidence to suggest that phytoestrogens found in cereal grains can reduce the risk of breast or prostate cancers.

\section{Maintain a healthy weight and be physically active}

Body Mass Index is a measure of body mass in relation to height (weight divided by the height squared). A Body Mass Index of 20-25 is considered healthy.

Ideally, one should aim for an hour's moderate or half an hour's vigorous exercise a day, but any increase in exercises is likely to be beneficial in proportion to the size of the increase. Brisk walking constitutes moderate exercise, running vigorous exercise.

\section{Drink alcohol in moderation if at all}

Any alcoholic drink irrespective of type or concentration increases the risk of cancer. However, in light of the beneficial effects small amounts of alcohol have on risk of cardiovascular disease, the Cancer Council supports prudent public health guidelines on alcohol consumption, namely no more than four standard drinks a day for men and two standard drinks a day for women. A standard drink contains 10 grams of alcohol.

\section{Select foods low in salt and fat}

Diets high in salt increase the risk of stomach cancer. Many processed foods are high in salt with consumption rates particularly high among young men.

Diets high in fat increase the risk of obesity and this increases the risk of endometrial cancer.

Diets high in saturated fats possibly increase the risk of colorectal, lung and prostate cancers, although the evidence is not at all conclusive.

For good health and to reduce the risk of obesity it is advised that people limit their saturated fat intake and opt for limited use of mono- and polyunsaturated fats.

There is a strong correlation between intake of saturated fat and intake of meat. While there is some evidence that eating red meat per se may increase the risk of colorectal cancer, no certain conclusion can be drawn about this association, or the possibility that eating 'browned' (that is, chargrilled) meat increases the risk of colorectal cancer. 


\section{POTENTIAL EFFECT ON CANCER OF IMPROVED DIET AND INCREASED EXERCISE}

The Australian Institute of Health and Welfare has estimated that 9.2 per cent of cancer deaths in 1996 were attributable to an intake of less than five servings a day of fruit and vegetables, 6.6 per cent were due to lack of any vigorous physical activity, 3.0 per cent were due to having a body mass index of 25 or more, and 3.6 per cent were due to intake of alcohol at any level. ${ }^{2}$ Since these estimates were generally conservative and do not include all possible sources of dietary or energy balance impact on cancer risk, the resulting total estimate, 22 per cent of cancer deaths attributable to diet or lack of exercise, is probably a minimum estimate.

This estimate accords reasonably well, therefore, with other estimates of 30-40 per cent of cancer deaths being preventable by dietary and related means.

\section{CONCLUSION}

Dietary and related energy balance factors have an important effect on cancer incidence and mortality in Australia. While the estimated effect is not as great as that of tobacco smoking (26 per cent of cancer deaths in 1996 due to smoking, as estimated by the Australian Institute of Health and Welfare), ${ }^{2}$ the real effect may be as great or greater. Therefore, effective implementation of the Cancer Council guidelines for prevention of cancer by diet and exercise, or similar guidelines, would be expected to yield substantial benefit.
Moreover, these guidelines generally sit well with more general dietary advice, which is designed to reduce the risk of cardiovascular disease and diabetes as well. It is time that improved diet and increased exercise were more consistently and effectively promoted by public health agencies in Australia.

\section{REFERENCES}

1. Doll R, Peto R. The causes of cancer-quantitative estimates of avoidable risks of cancer in the United States today. J Natl Cancer Inst 1981; 66:1195-1308.

2. World Cancer Research Fund and the American Institute for Cancer Research. Food, Nutrition and the Prevention of Cancer: a Global Perspective. New York: American Institute for Cancer Research, 1997.

3. Working Group on Diet and Cancer of the Committee on Medical Aspects of Food and Nutrition Policy. Nutritional Aspects of the Development of Cancer. London: The Stationery Office. 1998.

4. Mathers C, Vos T, Stevenson C. The burden of disease and injury in Australia. Canberra: Australian Institute of Health and Welfare, 1999.

5. Slavin J, Jacobs D, Marquart L. Whole-grain consumption and chronic disease: protective mechanisms. Nutr Cancer 1997; 27:14-21.

6. National Health and Medical Research Council. The prevention, early detection and management of colorectal cancer. Canberra: Australian Government Publishing Service, 1999.

7. National Health and Medical Research Council. Acting on Australia's weight: a strategic plan for the prevention of overweight and obesity. Canberra: Australian Government Publishing Service, 1997.

\section{INCREASING THE CONSUMPTION OF VEGETABLES AND FRUIT: A NATIONAL PUBLIC HEALTH CALLTO ACTION}

Philip Vita, Jane Moxon and Edwina Macoun Health Promotion Branch

NSW Department of Health

There is a need for national public health action to increase fruit and vegetable consumption for the protection and prevention of chronic disease, including many cancers. There has never been a better time to advance public health nutrition in Australia. The impetus has been provided by the Strategic Inter-Governmental Nutrition Alliance (SIGNAL) through the National Public Health Partnership, which has developed a national framework for action in public health nutrition EAT WELL Australia: An agenda for action for public health nutrition 20002010. As part of this, SIGNAL has developed a National Action Plan to promote the consumption of vegetables and fruit. ${ }^{1}$ This articles outlines the key components of the National Action Plan, and the role of the NSW Department of Health to increase vegetable and fruit consumption to ensure that the effect of diet-related disorders, including cancer, is reduced.

\section{BACKGROUND}

There is compelling evidence that consumption of vegetables and fruit offers protection against many cancers, including colorectal and stomach cancers. ${ }^{2}$ The challenge for public health is to develop effective and sustainable initiatives. Dietary behaviour is very complex and not well understood. Diet is influenced by a myriad of individual, social, economic and environmental factors. Moreover, it is inherently difficult to monitor-and consequently to assess-change. To improve population health through dietary interventions it is important to consider the supply-demand balance for food products in our community (Figure 6). It is crucial that policies and programs concurrently increase consumer demand for healthier foods, as well as address structural barriers to access and supply. ${ }^{3}$ 